\title{
Aberrated Microlenses to Reduce Crosstalk in Free Space Optical Interconnects Systems
}

\author{
Nedal Al-ababneh ${ }^{1}$ \\ ${ }^{1}$ Department of Electrical Engineering, Jordan University of Science and Technology, Irbid, Jordan \\ Correspondence: Nedal Al-ababneh, Department of Electrical Engineering, Jordan University of Science and \\ Technology P.O. Box 3030, Irbid 22110, Jordan. Tel: 962-720-1000, Ext. 22552. E-mail: nedalk@just.edu.jo
}

Received: April 8, 2018

doi:10.5539/mas.v12n5p100

\author{
Accepted: April 16, 2018 \\ Online Published: April 30, 2018 \\ URL: https://doi.org/10.5539/mas.v12n5p100
}

\begin{abstract}
A free space optical interconnects system that uses Gaussian beams and focusing microlenses with spherical aberration has been considered. Numerical results show that the use of microlenses with negative spherical improves the signal-to-crosstalk ratio of the interconnects system at some optimum positions of the detector array. An expression for output field at the detectors plane is derived and used to evaluate the signal-to-crosstalk ratio.
\end{abstract}

Keywords: lens spherical aberration, free space optical interconnects, optical crosstalk

\section{Introduction}

Microlenses (MLs) and microlenses arrays have become indispensable micro optical devices in free space optical interconnects (FSOIs) systems [Wang, Nirmalathas, 2018]. These MLs are used to gather the light and to focus it on a detector array. However, optical beams that propagate through MLs experience some deteriorations in their transverse profile due to their finite aperture and lens aberration [Hu, et.al., 2010]. In fact, the diffracted light from the MLs apertures, in these arrayed multi-channel micro optical systems, sum up in victim channel and cause crosstalk at the detector array [Al-Ababneh, 2014]. The MLs aberration also contribute the crosstalk by changing the focus point of the beam or by moving the beam on the detector array.

Many research papers have been done on the effect of MLs apertures on the quality of the propagating beams and many different models to evaluate the crosstalk in the presence of these finite apertures were introduced [Alkelly, et. al., 2011]. In [Al-Ababneh, 2014], analytical models to evaluate the optical crosstalk taking the effect of ML aperture were proposed. Using these models, the impact of different parameters on the optical system performance was addressed for minimum crosstalk. The use of MLs with Gaussian transmittance to reduce the crosstalk was proposed in [Al-Ababneh, 2014].

The aberrations of MLs and their effect in optical systems were also considered in the literature. The influence of many aberration types of the MLs on the propagation of the Gaussian beams used to carry the data in FSOIs system is considered. For example, in [Al-Ababneh, 2016] the impact of the spherical aberrations on the spot size and the light intensity for Gaussian beam passing through a free space optical interconnects is explained. As a general conclusion in these research papers the impact of the aberration is undesired as these aberrations cause shift, tilt, or defocus of the light beam at the observation plane.

In this paper we show that MLs aberrations can be exploited to reduce the crosstalk in FSOIs systems that use spherically aberrated ML. In fact, the effect of the lens with negative spherical aberrations (SA) on the propagated beam is to shift the beam focus toward the detector array. In this case the propagated focused beam will not spread that much and less overlap with neighbor detectors. Compared to other methods used to reduce crosstalk, the main advantage of this method is that it is lossless and simple.

To best of the author' knowledge, the use of MLs with negative SA to reduce crosstalk in FSOIs systems has not yet been investigated. In section 2 the optical field at the detector plane for the FSOIs system assuming microlenses with negative SA is evaluated. Performance evaluation of the FSOIs system showing the effect of SA is considered in section 3. Numerical results are introduced in section 4. Section 5 concludes the paper.

\section{Optical Field Distribution in Presence of Negative SA in FSOI System}

Figure 1 shows a simple schematic of the FSOIs system we consider in this paper. This optical system consists 
of light sources array, microlenses array, and detectors array. The distance between the light sources and the detectors arrays is $d_{1}$. The distance between the microlenses and the detectors array is $d_{2}$. The microlenses have circular apertures with uniform transmission profiles.

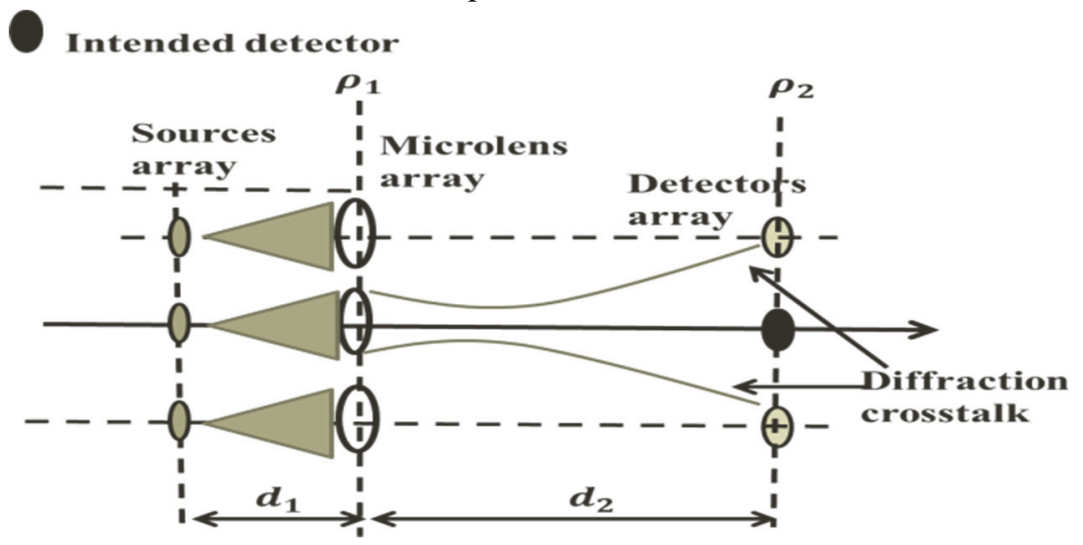

Figure 1. Schematic of FSOIs system

For ideal microlenses without SAs, the optical field at the detector plane where $z=\rho_{2}$ can be given by [Collins, 1970]

$$
\begin{aligned}
E_{2}\left(\rho_{2}, \theta_{2}\right) & =\frac{i k}{2 \pi b} \int_{0}^{\infty} \int_{0}^{2 \pi} E_{1}\left(\rho_{1}, \theta_{1}\right) A_{p}\left(\rho_{1}\right) \exp \left[-\frac{i k a}{2 b} \rho_{1}^{2}\right] \\
& \times \exp \left[-\frac{i k}{2 b} d \rho_{2}^{2}\right] \times \exp \left[\frac{i k \rho_{1}}{b} \rho_{2} \cos \left(\theta_{1}-\theta_{2}\right)\right] \rho_{1} d \rho_{1} d \theta_{1}
\end{aligned}
$$

$\rho_{1}, \theta_{1}$ and $\rho_{2}, \theta_{2}$ are the cylindrical coordinates at the plane $z=\rho_{1}$ and the plane $z=\rho_{2}$, respectively. $A P\left(\rho_{1}\right)$ is the aperture function. $k=2 \pi / \lambda$ is the wave number and $\lambda$ is the beam wavelength. The parameters $a, b, c$, and $d$ are the transfer matrix elements of the free space optical system and are given as:

$$
\left[\begin{array}{ll}
a & b \\
c & d
\end{array}\right]=\left[\begin{array}{cc}
1-\left(d_{2} / f\right) & 1-\left(d_{2} / f\right) d_{1}+d_{2} \\
-1 / f & 1-\left(d_{1} / f\right)
\end{array}\right]
$$

$E_{1}\left(\rho_{1}, \theta_{1}\right)$ is the field distribution at the plane $z=\rho_{1}$. Assuming Laguerre Gaussian model, $\mathrm{E}_{1}\left(\rho_{1}, \theta_{1}\right)$ can be given by

$$
\mathrm{E}_{1}\left(\rho_{1}, \theta_{1}\right)=\exp \left[-\frac{\rho_{1}^{2}}{\omega_{1}^{2}}\right]
$$

$\omega_{1}$ is the beam radius and is given by

$$
\omega_{1}=\omega_{0} \sqrt{1+\frac{\lambda^{2} f_{t}^{2}}{\pi^{2} \omega_{0}^{4}}}
$$

$\omega_{0}$ is the waist radius of the beam emitted from the light source. For circular aperture with radius of $a_{1}$, $A_{p}\left(\rho_{1}\right)$ can be written as

$$
A_{p}\left(\rho_{1}\right)= \begin{cases}1 & \rho_{1} \leq a_{1} \\ 0 & \rho_{1} \succ a_{1}\end{cases}
$$

For microlenses with negative SA, a phase factor $A_{b}\left(\rho_{1}\right)$ [González-Galicia,et.al., 2011] is used to describe the lens aberration as:

$$
A_{b}\left(\rho_{1}\right)=\exp \left[-i k \phi\left(\rho_{1}\right)\right]
$$


$\phi\left(\rho_{1}\right)$ is the microlens SA function and is given by

$$
\phi\left(\rho_{1}\right)=C_{1} \rho_{1}^{4}
$$

$C_{1}$ is the SA coefficient. Substituting Eq. (3), Eq. (5), Eq. (6), and Eq. (7), Eq. (1) can be written as

$$
\begin{aligned}
E_{2}\left(\rho_{2}, \theta_{2}\right) & =\frac{i k}{2 \pi b} \int_{0}^{a_{1}} \int_{0}^{2 \pi} \exp \left[-i k C_{1} \rho_{1}^{4}\right] \exp \left[-\frac{\rho_{1}^{2}}{\omega_{1}^{2}}-\frac{i k a}{2 b} \rho_{1}^{2}\right] \\
& \times \exp \left[-\frac{i k}{2 b} d \rho_{2}^{2}\right] \times \exp \left[\frac{i k \rho_{1}}{b} \rho_{2} \cos \left(\theta_{1}-\theta_{2}\right)\right] \rho_{1} d \rho_{1} d \theta_{1}
\end{aligned}
$$

Eq. (8) provides a convenient expression to evaluate the optical field distribution at the detector plane in the FSOIs system using microlenses with SA. In this case, the light intensity distribution can be given as

$$
I_{2}\left(\rho_{2}, \theta_{2}\right)=\left(\frac{i k}{2 \pi b}\right)^{2}\left|\int_{0}^{a_{1}} \int_{0}^{2 \pi} \exp \left[i k C_{1} \rho_{1}^{4}\right] \exp \left[-\frac{\rho_{1}^{2}}{\omega_{1}^{2}}-\frac{i k a \rho_{1}^{2}}{2 b}\right] \exp \left[\frac{i k \rho_{1} \rho_{2}}{b} \cos \left(\theta_{1}-\theta_{2}\right)\right] \rho_{1} d \rho_{1} d \theta_{1}\right|^{2}
$$

Equation (9) will be used to show the effect of negative SA on the signal-to-crosstalk ratio in the next section.

\section{Signal-To-Crosstalk Ratio}

To estimate the signal-to-crosstalk ratio, two types of optical crosstalk are evaluated. The first type of crosstalk is diffraction crosstalk which results from the light coupled to the intended detector from the diffracted beams of the other light sources through the other microlenses. The diffraction crosstalk power received by the intended detector can be found as the power received by all neighboring detectors from the light coming through intended microlens assuming only the intended light source is on. Therefore, the diffraction crosstalk power can be given as

$$
P_{n d}=4 \iint_{\psi_{1}} \mathrm{I}_{2}\left(\rho_{2}, \theta_{2}\right) \rho_{2} d \rho_{2} d \theta_{2}+4 \iint_{\psi_{2}} \mathrm{I}_{2}\left(\rho_{2}, \theta_{2}\right) \rho_{2} d \rho_{2} d \theta_{2}
$$

$\psi_{1}$ and $\psi_{2}$ are the areas covered by one of the neighbor and one of the next neighbor detectors, respectively. The second type is the stray light crosstalk [Tang, et al., 1994]. The stray crosstalk power is the total power received by all neighboring detectors from the light coming through neighboring microlenses assuming only the intended light source is on. The stray crosstalk power can be given as

$$
P_{n s}=4 \iint_{\psi_{3}}\left|\mathrm{E}_{1}\left(\rho_{1}, \theta_{1}\right) \cdot A_{p}\left(\rho_{1}\right)\right|^{2} \rho_{1} d \rho_{1} d \theta_{1}+4 \iint_{\psi_{4}}\left|\mathrm{E}_{1}\left(\rho_{1}, \theta_{1}\right) \cdot A_{p}\left(\rho_{1}\right)\right|^{2} \rho_{1} d \rho_{1} d \theta_{1}
$$

$\psi_{3}$ and $\psi_{4}$ are the areas covered by one of neighbor and one of the next neighbor microlenses, respectively. The signal power is the power received by the intended detector from the light that reaches the intended light source through the intended microlens. Therefore, the signal power can be given by:

$$
P_{\text {sig }}=\iint_{\psi} \mathrm{I}_{2}\left(\rho_{2}, \theta_{2}\right) \rho_{2} d \rho_{2} d \theta_{2}
$$

$\psi$ is the area of the intended detector. Having evaluated the signal and the crosstalk power, the signal to crosstalk (SCR) for the FSOIs can be determined from:

$$
S C R=\frac{P_{s i g}}{P_{n c}+P_{n s}}
$$

\section{Results}

In this section numerical simulations are introduced to show the improvement on the SCR that can be obtained using microlenses with negative SA. In these simulations, the light source has a wavelength $\lambda=0.85 \mu \mathrm{m}$ and a beam waist radius of $\omega_{0}=3 \mu \mathrm{m}$. The radius of the microlens is $125 \mu \mathrm{m}$ and its focal length is $720 \mu \mathrm{m}$. The 
distances $d_{2}=2.5 \mathrm{~mm}$ and $d_{1}=f$. The length of optical interconnect is $l=3.22 \mathrm{~mm}$ and the interconnect pitch is $250 \mu \mathrm{m}$. Figure 2 shows the SCR versus detector radius with and without SA. The values of the aberration coefficient are $C_{1}=0,1.116$ and $-1.116 \mathrm{~mm}^{-3}$. It is clear from the figure that the maximum SCR is 30 using the microlens of negative SA, 22.6 for no aberration, and 19.2 for positive SA. Furthermore, the optimum detector radius in case of negative SA is smaller than that of no aberration and positive SA cases. For maximum SCR, the optimum detector radii for no aberration, positive SA, and negative SA are 25 $\mu \mathrm{m}, 23 \mu \mathrm{m}$, and $21 \mu \mathrm{m}$, respectively. The change of SCR in the presence of SA can be explained by considering figure 3 .

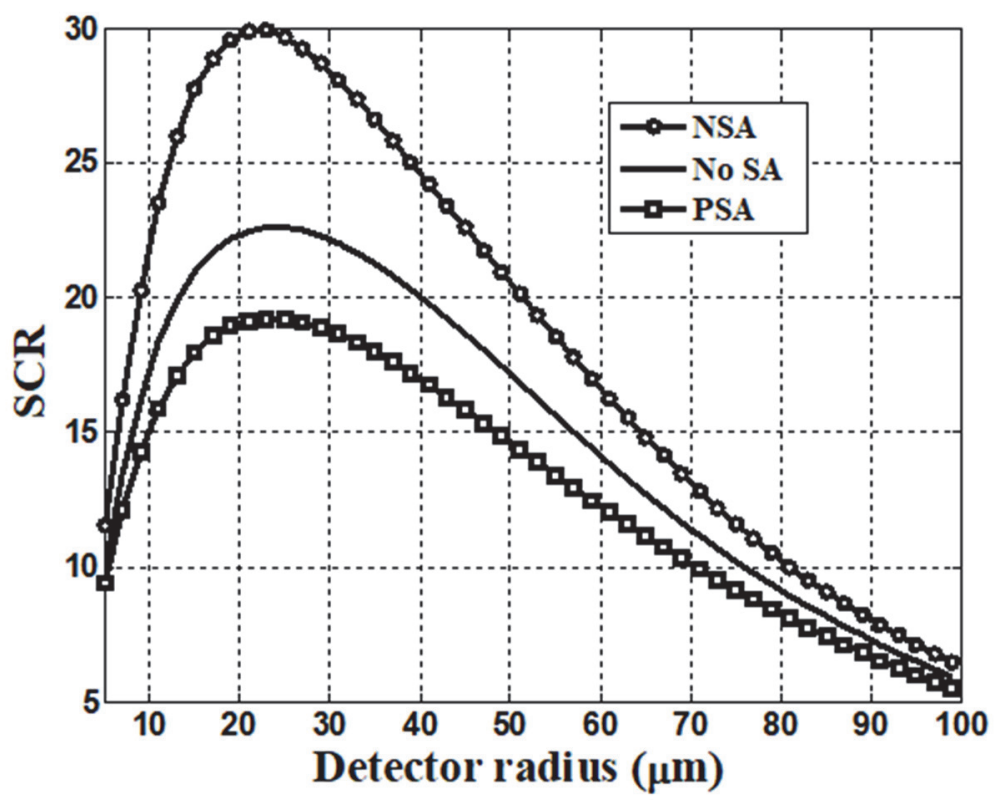

Figure 2. Signal-to-crosstalk ratio versus detector radius with and without SA

In figure 3 we have plotted the normalized crosstalk power versus detector radius. It is clear from the figure that the crosstalk power in the presence of negative SA is smaller than that of no aberration and positive SA cases. The decrease of the crosstalk in presence of negative SA can be explained by careful examination of figures 4 and5. Figure 4 shows the normalized intensity distribution at the detector plane with and without SA. It is seen from the figure that the lens aberration changes the optical distribution of the beam by changing the peak axial intensity and the light intensity in the transverse direction. In this case, the presence of negative aberration results in a decrease in the cross power and an increase in the signal power. 


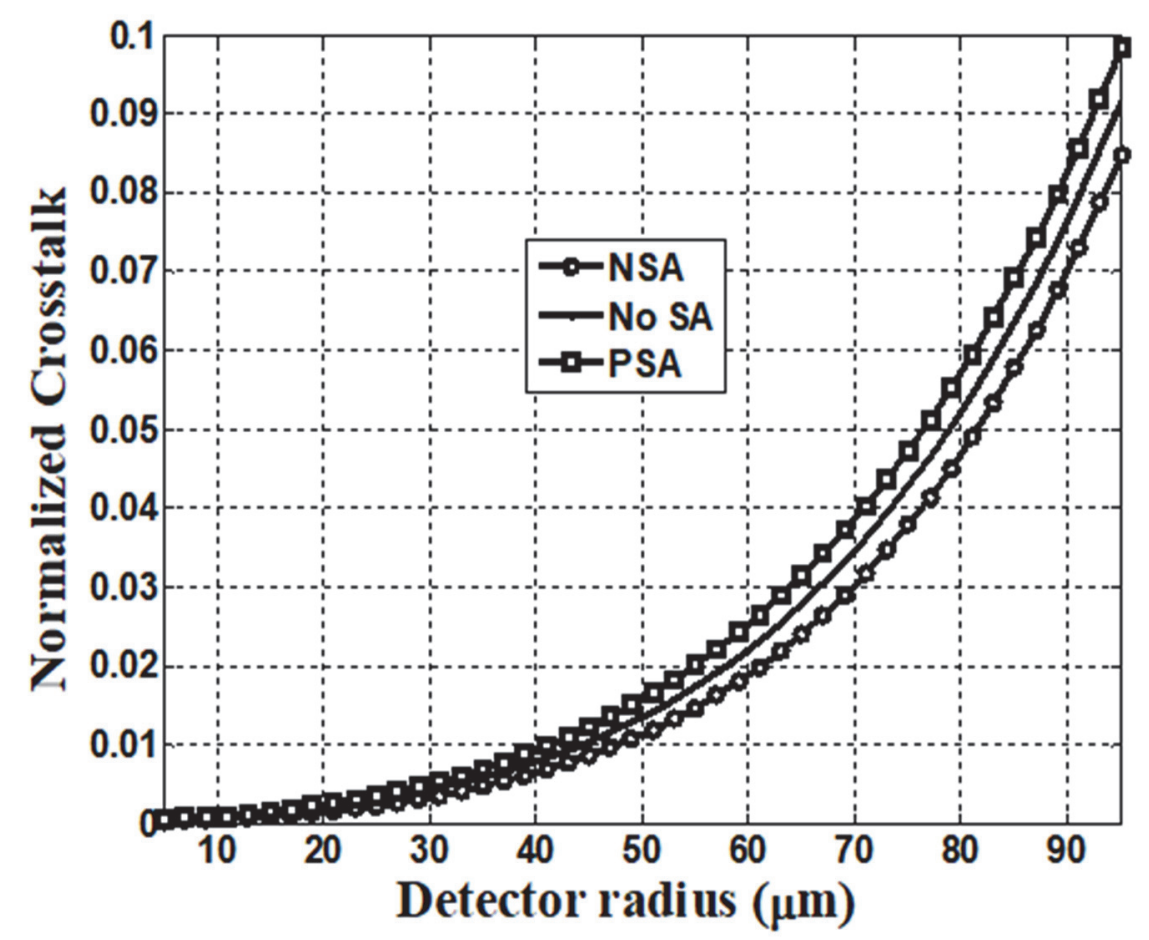

Figure 3. Normalized crosstalk versus detector radius with and without SA

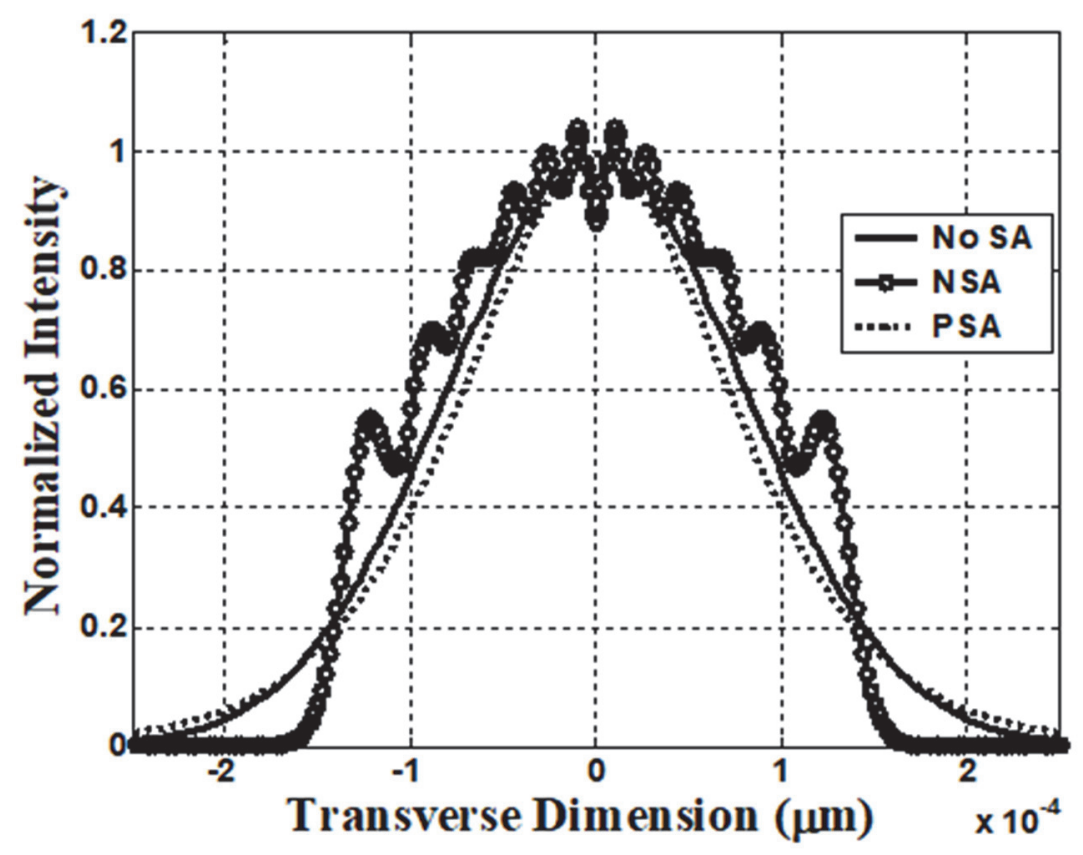

Figure 4. Normalized intensity distribution at the detector plane with and without SA

Figure 5 shows the normalized (with respect to no aberration case) maximum axial intensity versus interconnect length with and without lens aberration. It is noted from the figure that the peak intensity of the beam occurs before the focus point of the lens in the case of negative SA. In this case, the beam will spread more as it propagates toward the detector array which is located beyond the focus point. In fact, these observations manifest in figure 5 . 


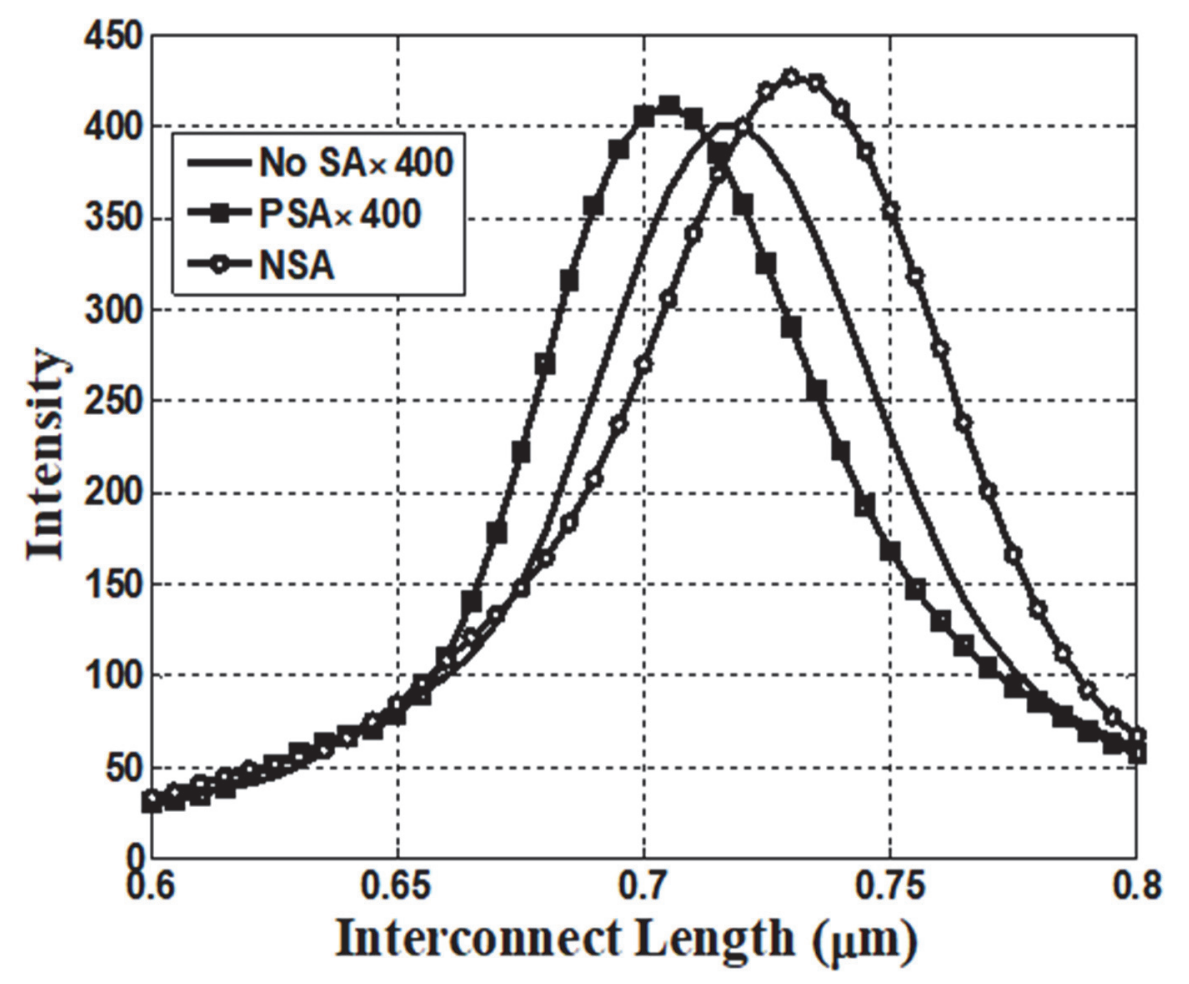

Figure 5. Normalized peak intensity versus interconnect length with and without SA

\section{References}

Al-Ababneh, N. (2014). Crosstalk reduction in free space optical interconnects systems using microlenses with Gaussian transmittance. Optics Communications, 318, 79-82. https://doi.org/10.1016/j.optcom.2013.12.063

Al-Ababneh, N. (2016). Crosstalk in Free Space Optical Interconnects that Use Micro-Lenses Arrays: Practical Consideration. In Applied Mechanics and Materials (Vol. 850, pp. 95-99). Trans Tech Publications. https://doi.org/10.4028/www.scientific.net/AMM.850.95

Al-Ababneh, N. K. (2014). Approximate analytical method to evaluate diffraction crosstalk in free-space optical interconnects systems that use circular microlenses with finite uniform apertures. Optical Engineering, 53(7), 076104. https://doi.org/10.1117/1.OE.53.7.076104

Alkelly, A. A., Al-Nadary, H., \& Alhijry, I. A. (2011). The intensity distribution of hollow Gaussian beams focused by a lens with spherical aberration. Optics communications, 284(1), 322-329. https://doi.org/10.1016/j.optcom.2010.08.040

Collins, S. A. (1970). Lens-system diffraction integral written in terms of matrix optics. JOSA, 60(9), 1168-1177 https://doi.org/10.1364/JOSA.60.001168.

Hu, W., Li, X., Yang, J., \& Kong, D. (2010). Crosstalk analysis of aligned and misaligned free-space optical interconnect systems. JOSA A, 27(2), 200-205. https://doi.org/10.1364/JOSAA.27.000200

Wang, K., Nirmalathas, A., Lim, C., Wong, E., Alameh, K., Li, H., \& Skafidas, E. (2018, March). 80 Gb/s FreeSpace Reconfigurable Optical Interconnects with Carrierless-Amplitude-Phase Modulation and Space-Time Block Code. In Optical Fiber Communication Conference (pp. M2K-6). Optical Society of America. https://doi.org/10.1364/OFC.2018.M2K.6

\section{Copyrights}

Copyright for this article is retained by the author(s), with first publication rights granted to the journal.

This is an open-access article distributed under the terms and conditions of the Creative Commons Attribution license (http://creativecommons.org/licenses/by/3.0/) 